\title{
Video Abstracts
}

\section{Parkinsonism and Tremor in a Patient with Plasmodium vivax Malaria}

\author{
Jung E. Park*
}

Department of Neurology, Dongguk University Ilsan Hospital, Goyang, Republic of Korea

\begin{abstract}
Background: A 57-year-old male diagnosed with Plasmodium vivax malaria presented with a subacute onset of hand tremor, slowness, and gait difficulty. Phenomenology: A bilateral upper extremity kinetic tremor was seen, as well as a right upper extremity rigidity and body bradykinesia.

Educational Value: Parkinsonism and tremor are neurological manifestations that may occur in malaria as a result of globus pallidi and dentate nuclei involvement.
\end{abstract}

Keywords: Parkinsonism, tremor, malaria, cerebral, Plasmodium vivax

Citation: Park JE. Parkinsonism and tremor in a patient with Plasmodium vivax malaria. Tremor Other Hyperkinet Mov. 2017; 7. doi: 10.7916/D8P55V23

*To whom correspondence should be addressed. E-mail: noizegun@gmail.com

Editor: Elan D. Louis, Yale University, USA

Received: April 26, 2017 Accepted: May 16, 2017 Published: June 5, 2017

Copyright: $\odot 2017$ Park. This is an open-access article distributed under the terms of the Creative Commons Attribution-Noncommercial-No Derivatives License, which permits the user to copy, distribute, and transmit the work provided that the original authors and source are credited; that no commercial use is made of the work; and that the work is not altered or transformed.

Funding: None.

Financial Disclosures: Dr. Park has received research grants from the Dystonia Medical Research Foundation, SK chemicals, and the National Research Foundation of Korea.

Conflicts of Interest: The author reports no conflict of interest.

Ethics Statement: All patients that appear on video have provided written informed consent; authorization for the videotaping and for publication of the videotape was provided.

A 57-year-old male presented with hand tremor, bradykinesia, and gait difficulty. The patient visited an emergency room with initial symptoms of persistent fevers and myalgia. Temperature was $39.1^{\circ} \mathrm{C}$, blood pressure 85/54, heart rate $129 \mathrm{bpm}$, respiratory rate 18 and $99 \% \mathrm{SpO}_{2}$. Laboratory results were notable for a mild anemia (hemoglobin of $11.7 \mathrm{~g} / \mathrm{dL}$; reference values $13-17 \mathrm{~g} / \mathrm{dL}$ ), thrombocytopenia (platelet count of 35,000; reference values $150-400 \times 10^{3} / \mu \mathrm{L}$ ), and elevated transaminase (aspartate aminotransferase 42; reference values $<40$, alanine aminotransferase 59 ; reference values $<41$ ). As he lived in an endemic area for malaria (Kyong-gi province) and engaged in gardening, serological testing for malaria antibodies (Abs) and antigens (Ags) (i.e., Plasmodium falciparum, Plasmodium vivax) was performed, which was positive for $P$. vivax $\mathrm{Ag}$ and Ab. Microscopy of a peripheral blood smear confirmed the presence of $P$. vivax. He was diagnosed with malaria and completed a 2-week course of chloroquine 2,000 $\mathrm{mg}$ and primaquine $26.3 \mathrm{mg}$ every day.

The patient presented to our institution approximately 4 weeks following treatment of malaria with symptoms of hand tremor, slowness, and gait difficulty (Figure 1). He stated that these symptoms had developed during the course of treatment for malaria. On neurological examination, hypomimia and hypophonia were noted. Tone was increased in the right upper extremity, and a bilateral upper extremity kinetic tremor was seen. Mild limb bradykinesia was noted on finger tapping in the right upper extremity and on opening and closing of both hands. Decreased right arm swing and en-bloc turning was noted on gait and postural instability. The Unified Parkinson Disease Rating Scale part III was 11 (Video 1).

Brain magnetic resonance imaging revealed hyperintensities of bilateral globus pallidi on fluid-attenuated inversion recovery (FLAIR). Hyperintensities in the bilateral dentate nuclei of the cerebellum were also seen on T2 and FLAIR sequences. The patient was started on carbidopa-levodopa 25-100 mg three times a day, and experienced mild improvement of body bradykinesia.

\section{Discussion}

We report a case of Parkinsonism and tremor in a patient diagnosed with malaria. $P$. vivax is the main etiology for malaria in South Korea, mostly present along the demilitarized zone. ${ }^{1}$ Our patient's initial presentation of fevers and myalgia is consistent with the clinical picture of malaria. ${ }^{2}$ The hand tremor, slowness and gait difficulty however, is an unusual presentation. Cerebral malaria is a rare complication $(2.4 \%$ incidence in $P$. vivax infection), ${ }^{1,3}$ which can result in cerebral edema and increased mortality, ${ }^{3}$ but Parkinsonism has not been previously reported. Cerebral malaria has been reported to result in diffuse edema and less commonly, bithalamic and cerebellar lesions in 

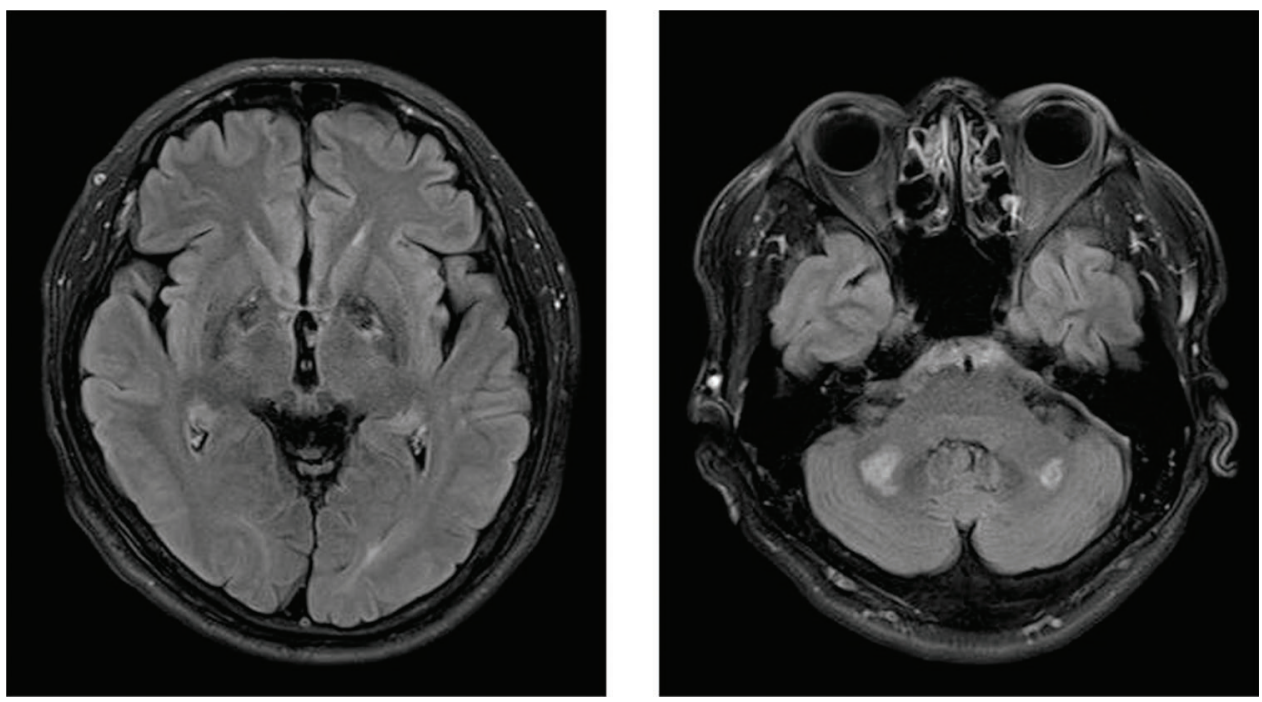

Figure 1. Magnetic Resonance Imaging of the Brain: Fluid-attenuated Inversion Recovery Sequence. Hyperintensities are seen in bilateral globus pallidi (left). Hyperintense lesions are noted in bilateral dentate nuclei (right).

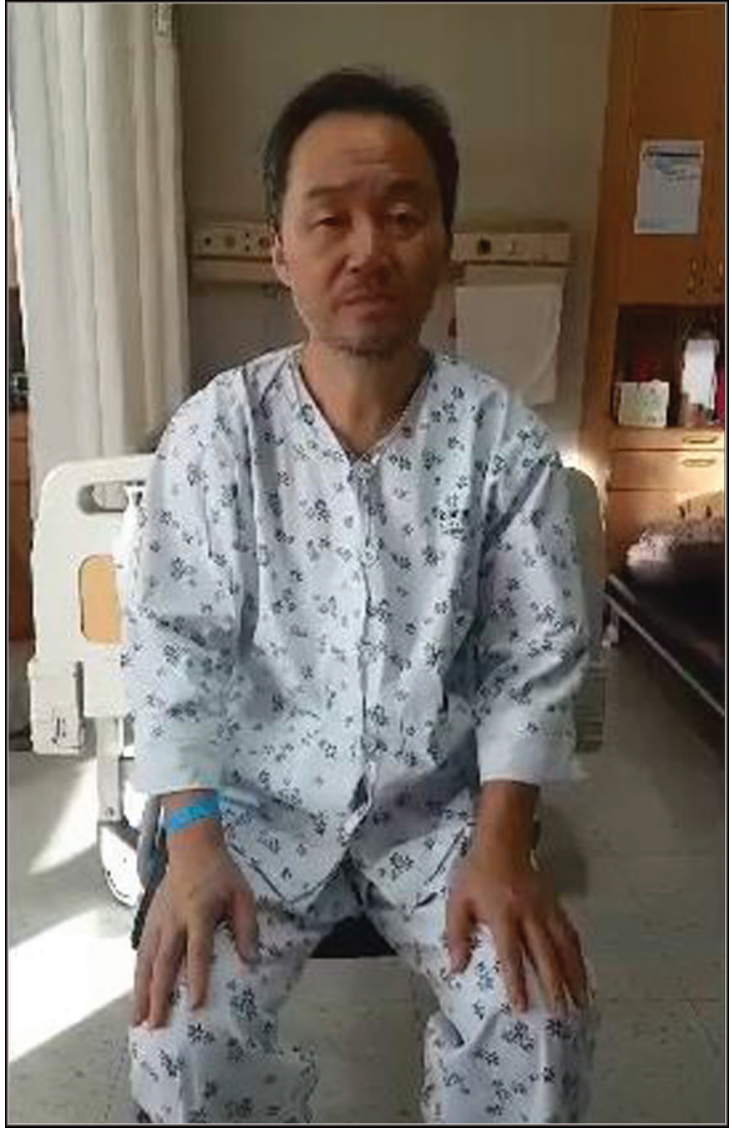

Video 1. Patient on Initial Visit to our Institution. Hypomimia and hypophonia are noted, as well as a kinetic tremor of both upper extremities. Body bradykinesia and en-bloc turning is seen on casual gait. Postural instability is noted on the pull test.
P. falciparum malaria. ${ }^{3}$ In our patient, globus pallidi and cerebellar involvement may have occurred as a result of hypoxic-ischemic injury. We presume that the globus pallidi lesions resulted in the Parkinsonian features including bradykinesia, rigidity and postural instability. The hand tremor, however, is likely secondary to dentate nuclei involvement, as this was seen as a largely symmetric kinetic tremor, not consistent with the resting tremor seen in Parkinsonism.

\section{References}

I. Im JH, Kwon HY, Baek J, et al. Severe Plasmodium vivax infection in Korea. Malar f 2017;16:51. doi: 10.1186/s12936-017-1684-4

2. Svenson JE, MacLean JD, Gyorkos TW, Keystone J. Imported malaria. Clinical presentation and examination of symptomatic travelers. Arch Intern Med 1995;155:861-868. doi: 10.1001/archinte.1995.00430080109013

3. Patankar TF, Karnad DR, Shetty PG, Desai AP, Prasad SR. Adult cerebral malaria: prognostic importance of imaging findings and correlation with postmortem findings. Radiology 2002;224:811-816. doi: 10.1148/radiol. 2243010588 